\title{
Photon Collection Efficiency of Fluorescent Solar Collectors
}

\author{
Pattareeya Kittidachachan, Lefteris Danos, Thomas J. J. Meyer, Nicolas Alderman, and Tom Markvart*
}

\begin{abstract}
Photon collection efficiency of fluorescent collectors is examined experimentally and by modelling, with a view to assess the potential of this attractive solar energy technology. The edge fluorescence of single dye collectors based on Rhodamine 6G and Coumarin 540A, manufactured by a simple fabrication technique, is investigated in detail. A novel technique for determination of the collection efficiency is presented based on the traditional model of fluorescent collectors but extended to allow spectral analysis. We show that these results agree well with direct solar cell measurements. General conclusions are presented about fundamental loss mechanisms and about the role of re-absorption of fluorescent light. It is shown that substantial improvements in collector efficiency can be made with the use of photonic fluorescence confinement rather than by total internal reflection.
\end{abstract}

Keywords: Fluorescent collectors · Photon collection efficiency · Solar energy

\section{Introduction}

Fluorescent concentrators (or collectors) for solar energy conversion have attracted interest for some time ${ }^{[1-6]}$ with the potential of concentrating the solar flux onto a smaller solar cell and thus reducing the total system cost. A typical fluorescent collector absorbs incident light through the front face of a luminescent plate. The luminescent light is trapped by total internal reflection (TIR) and guided to the edge of the plate and onto a solar cell (Fig. 1). One objective of the collector design is to reduce the size of the solar cell with respect to the area which collects light. Unlike their geometric counterparts fluorescent collectors can accept light from a wide angle of incidence and are thus able to make use of the diffuse light.

The luminescent medium can be organic dyes, inorganic absorbers or quantum dots embedded in a transparent medium such as polymethyl methacrylate (PMMA) or polycarbonate, glass or even liquid solutions. ${ }^{[7,8]}$ Several matrix materials with

E-Mail: tm3@soton.ac.uk

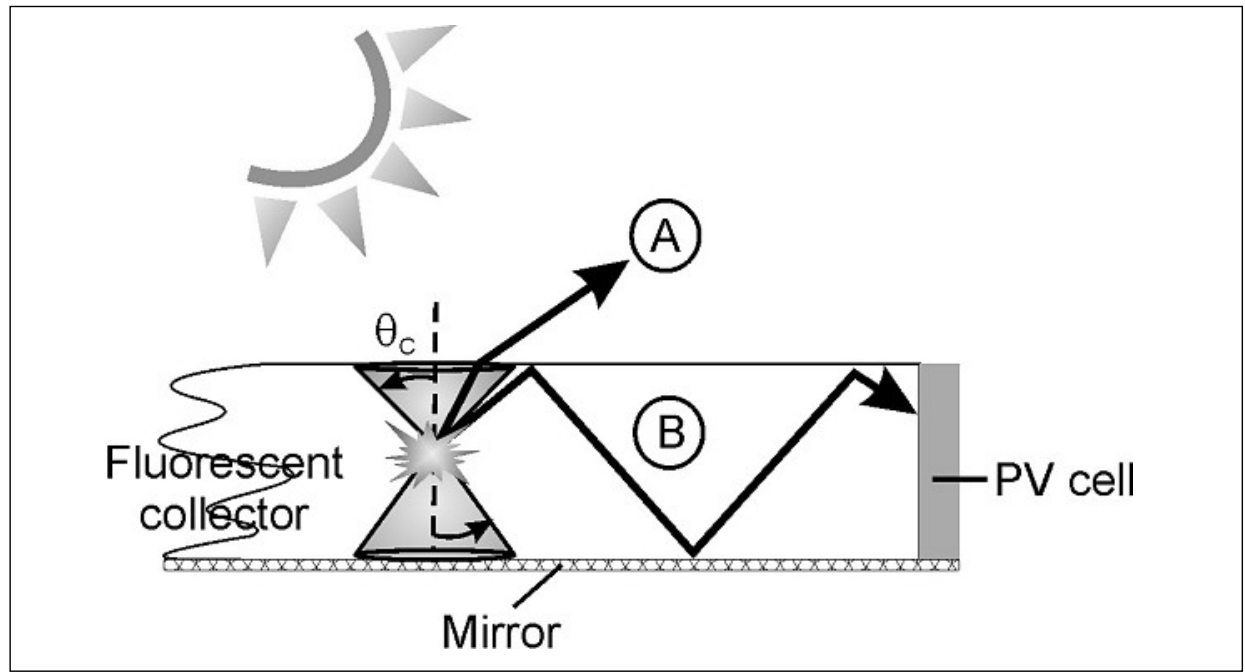

Fig. 1. Schematic diagram of fluorescent collector, showing the double escape cone with apex angle

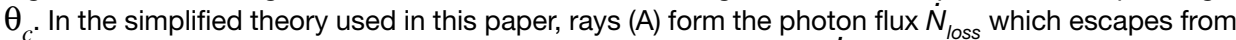
the front face of the collector, and rays (B) contribute to the flux $\dot{N}_{\text {work }}$ which illuminates the solar cell

similar refractive index can be used to make up the collector, dividing the roles of light absorption and photon transport. ${ }^{[4,9]}$

This paper presents an experimental and theoretical study of fluorescent collectors, focusing on photon collection efficiency, the equivalent of internal quantum efficiency of a solar cell. We report high collection efficiencies using a simple fabrication technique based on Rhodamine $6 \mathrm{G}$ dissolved in PMMA and deposited on glass as well as novel results obtained using liquid collectors based on Coumarin 540A.

The results are analysed using a traditional model ${ }^{[1,2,5,6]}$ which is extended to in- clude spectral dependence of the collection efficiency and compared with results obtained by ray tracing. This analysis permits an accurate assessment of re-absorption of fluorescent light (sometimes also called photon recycling $\left.{ }^{[10]}\right)$ in the collector and a convenient determination of the collection efficiency based on spectral analysis. We show that these results agree well with direct solar cell measurements.

We demonstrate that fluorescence reabsorption is an integral part of the operation of a fluorescent collector which allows the fluorescent light to attain thermal equilibrium with the fluorescent medium. 
General conclusions are presented about fundamental loss mechanisms using a generalisation of the traditional two-flux model. It is shown that substantial improvements in collector efficiency require the optical confinement to be based on spectral rather than geometric considerations. The implication is that new, highly efficient collectors could emerge whose operation will be based on photonic structures rather than light trapping by total internal reflection.

\section{Photon Balance in the Collector}

Let us denote by $\dot{N}_{\text {work }}$ the 'useful' luminescent photon flux (number of photons per unit time) which illuminates the solar cell, $\dot{N}_{\text {loss }}$ the photon flux emitted through the front surface which is lost from the system, and $\dot{N}_{a b s}$ the number of photons absorbed per unit time by the collector from the incident solar beam. The photon balance inside the collector can then be written as

$$
\dot{N}_{a b s}=\dot{N}_{l o s s}+\dot{N}_{w o r k}+\dot{N}_{n r}
$$

where, to maintain full generality, Eqn. (1) includes non-radiative de-excitation of the luminescent medium at the rate of $\dot{N}_{n r}$.

Traditional analyses of Weber and coworkers $^{[1,2]}$ and the Zewail group ${ }^{[5,6]}$ equate the useful photon flux $\dot{N}_{\text {work }}$ to the flux trapped within the collector by total internal reflection. The flux $\dot{N}_{\text {loss }}$ represents photons emitted within a double 'escape cone' with half apex angle of $\theta_{c}=\sin ^{-1}(1 / n)$, where $n$ is the refractive index of the collector material (Fig. 1).

Assuming isotropic emission by the fluorescent dyes and denoting by $P=1-\cos \theta_{c}$ the solid angle of the escape cone divided by $4 \pi$,

$$
\begin{aligned}
& \dot{N}_{\text {loss }}=\frac{1}{\tau_{\text {rad }}} P(1-\bar{R}) N^{*} \\
& \dot{N}_{\text {work }}=\frac{1}{\tau_{\text {rad }}}(1-P)(1-R) N^{*}
\end{aligned}
$$

where $\bar{R}$ and $R$ are the probabilities of re-absorption for photons within and outside the escape cone, respectively, $\tau_{r a d}$ is the radiative lifetime of the dye, and $N^{*}$ is the number of molecules in the excited state. Since

$$
\dot{N}_{n r}=\frac{1}{\tau_{n r}} N^{*}
$$

where $\tau_{n r}$ is the non-radiative lifetime, we find the fluorescence photon collection efficiency of the collector

$$
Q_{C}=\frac{\dot{N}_{w o r k}}{\dot{N}_{a b s}}=\phi_{f} \frac{(1-P)(1-R)}{1-\{(1-P) R-P \bar{R}\} \phi_{f}}
$$

where $\phi_{f}$ is the fluorescence quantum yield. Eqn. (4) has been derived by other means in a number of papers. ${ }^{[2,5]}$ If supplemented by the definition of the absorption efficiency $Q_{A}$,

$$
Q_{A}=\frac{\dot{N}_{a b s}}{I_{o}}
$$

where $I_{o}$ is the incident photon flux, in photons per unit time, the total optical efficiency is given by the compact expression

$$
\eta=\frac{\dot{N}_{\text {work }}}{I_{o}}=\frac{\dot{N}_{\text {work }}}{\dot{N}_{a b s}} \frac{\dot{N}_{a b s}}{I_{o}}=Q_{A} Q_{C}
$$

The overall photon balance expressed by Eqn. (1) gives a broad view of the concepts that are relevant to the operation of fluorescent collectors. To be of practical use, however, the photon collection efficiency needs to be specified as a function of wavelength - more precisely, as a function of the wavelength of the photons absorbed by the collector as well as photons which are incident on the solar cell. Since fluorescence is usually independent of the excitation wavelength, these two aspects can usually be considered separately.

The key component of the spectral analysis is the fluorescence spectrum of the dye which is free from re-absorption. Using the terminology of Zewail and Batchelder this spectrum will be called the first generation spectrum. Normalised to unit intensity, this spectrum will be denoted by $f_{l}(\lambda)$, with

$$
\int f_{1}(\lambda) d \lambda=1
$$

We can now define the spectral photon collection efficiency $\chi$,

$$
Q_{C}=\int \chi(\lambda) f_{1}(\lambda) d \lambda
$$

which, with the use of Eqn. (4), can be written as

$$
\chi(\lambda)=\frac{\phi_{f}(1-P)}{1-\{(1-P) R-P \bar{R}\} \phi_{f}}\{1-r(\lambda)\}
$$

Here, $r(\lambda)$ is the probability that a photon emitted at wavelength $\lambda$ outside the escape cone is re-absorbed. Clearly, we have

$$
R=\int r(\lambda) f_{1}(\lambda) d \lambda
$$

The above treatment is based on a number of simplifying assumptions, including a uniform distribution of the excited molecules throughout the collector, perfectly flat specular reflecting external surfaces, no scattering of light inside the collector, and no absorption of light except by the dye. It is also assumed that light emitted onto the solar cell leaves the collector within the full hemisphere, on account of the high refractive index of the semiconductor. Some of these features can be tackled analytically but a full treatment is likely to require the use of numerical techniques.

Following the treatment of Weber and Lambe, ${ }^{1]}$ the re-absorption probability $r(\lambda)$

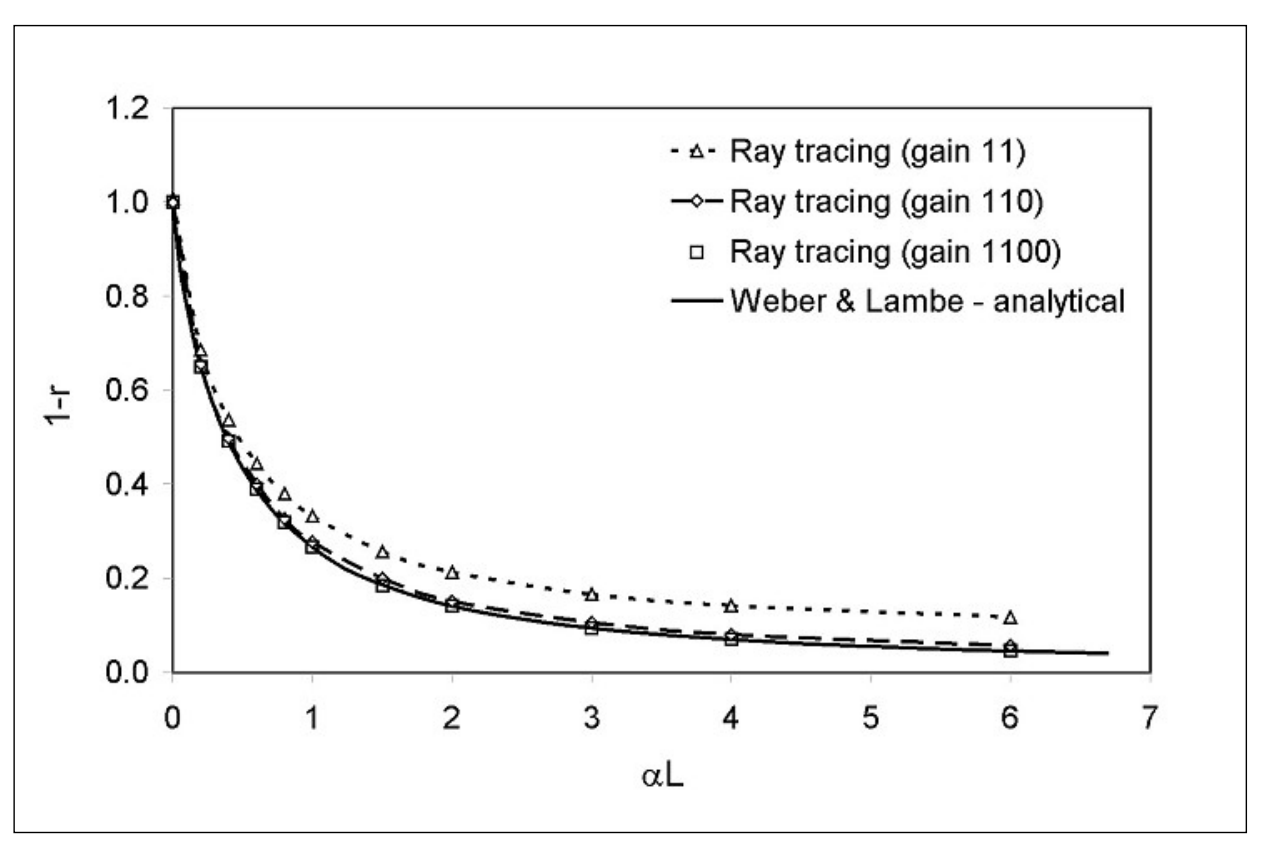

Fig. 2. Comparison of ray tracing results with the Weber and Lambe theory 
can easily be evaluated numerically. This is shown in Fig. 2 for a collector in the shape of an infinite strip of width $L$, with one edge covered by a solar cell and the opposite edge by a perfect mirror. Fig. 2 also shows the result obtained by ray tracing software (OptiCAD) for two geometric gain factors $G=A_{\text {fron }} / A_{\text {edge }}$ of the collector, where $A_{\text {front }}$ is the area of the illuminated front face of the collector and $A_{\text {edge }}$ is the area of the edge covered with the solar cell. Clearly, the Weber and Lambe approximation works well for collectors with a high gain but deviations occur when the gain factor is small.

\section{Experimental Details}

\subsection{Materials and Sample Preparation}

The fluorescent collectors were prepared by spin coating of dye-polymer solutions onto square glass substrates (Fig. 3a). This technique is similar to the method used by Rapp and Boling ${ }^{[4]}$ and produces thin polymer films onto glass substrates which are easy to fabricate. A solution of Rhodamine 6G(R6G, Acros Organics, 99\%) dye was prepared by diluting the dye with dichloromethane (DCM, Fisher Scientific) before mixing with Tensol 12 (RS) in an ultrasonic bath. In this study, the mixture ratio (Tensol:R6G solution) was fixed at $6: 2(\mathrm{w} / \mathrm{v})$. The prepared mixture was dropped along the glass slide (Menzel, $26 \mathrm{~mm} \times 26 \mathrm{~mm} \times 1 \mathrm{~mm}$, clear cut edges) and spin coated. The surface coverage of the substrate produced in this way was, to a good degree, uniform and reproducible to $\pm 10 \%$. The thickness of the films, measured by using a profilometer type Taylor-Hobson Form Talysurf-120 L (Rank Taylor Hobson Limited, Leicester, England), was found to be $9 \mu \mathrm{m}$. The dye concentration in the film was determined by integration of absorbance and comparison with a reference extinction coefficient of Rhodamine 6G in ethanol. The near-matching refractive index of the Tensol film (1.48) and glass slide (1.51, sodium Dline, $589 \mathrm{~nm}$ ) allowed the fluorescence emission to be wave-guided by the glass slide towards the edge.

For illustration of specific features of the collector spectroscopy, edge and front fluorescence spectra were also studied using liquid collectors in the shape of a thin custom-made glass cuvette with dimensions $l=50 \mathrm{~mm}, L=42 \mathrm{~mm}, d_{\text {liq }}=d_{\text {glass }}=0.75$ $\mathrm{mm}$, a side optical window and the inside edges of a gasket coated with an aluminium layer (Fig. 3b). The liquid collectors were prepared from ethanol (Fisher Scientific) solutions of Coumarin 540A (Exciton).

\subsection{Spectroscopic Measurements}

An Avantes spectrometer AvaSpec2048, grating UA (200-1100 nm), slit-25

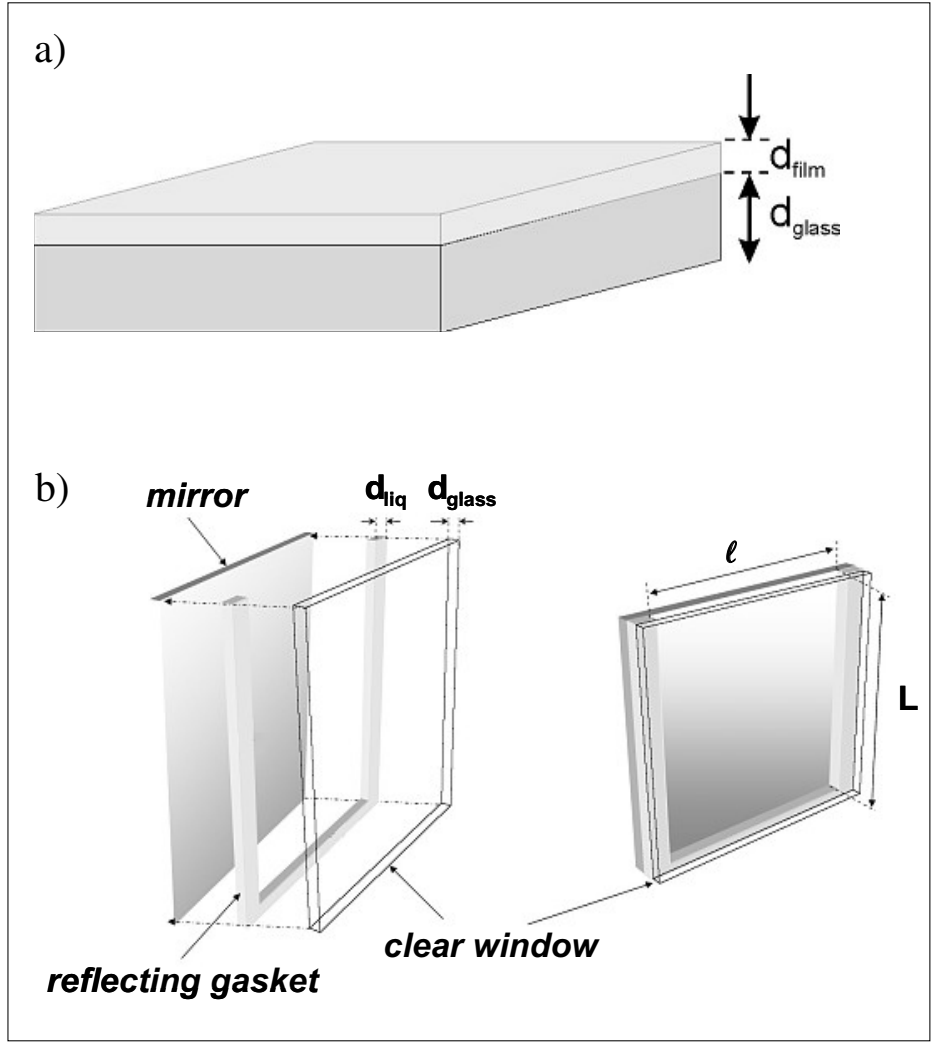

Fig. 3. A schematic diagram of solid (a) and liquid (b) collectors used in this work

$\mu \mathrm{m})$ based on the symmetrical CzernyTurner design with a 2048 pixel CCD detector array or a Bentham spectrometer equipped with a monochromator (TM300) and a PMT tube (Hamamatsu R446) or a calibrated silicon detector (dh_Si, Bentham Instruments Ltd) were used for all absorption and fluorescence measurements. Fibre optics was used to guide and collect the light to and from the collectors.

All absorption spectra (400-700 nm) were measured directly from Rhodamine 6G films spin coated on collectors shown in Fig. 3a. Since the refractive index of Tensol is similar to that of glass, the edge fluorescence spectra of these collectors were analysed by treating them as homogeneous plates, with an effective absorption coefficient of

$$
\alpha_{\text {eff }}=\alpha \frac{d_{\text {film }}}{d_{\text {film }}+d_{\text {glass }}} \cong \alpha \frac{d_{\text {film }}}{d_{\text {glass }}}
$$

where $\alpha$ is the true absorption coefficient of the dye-loaded PMMA film of thickness $d_{\text {film }}$, and $d_{\text {glass }}$ is the thickness of the glass slide. Eqn. (10) is justified by a simple geometrical consideration of the optical path of the beams emitted by the dye that reach the edge of the collector and are reflected - usually many times - from its front and rear surface. Solution absorption spectra (350-700 nm) of Coumarin 540A were measured directly from the liquid collectors.

The methodology discussed in this paper rests on an accurate determination of the fluorescence spectra which are free from re-absorption effects. ${ }^{[11]}$ For liquid collectors, the determination of first-generation spectra is a simple matter as they can be obtained by standard methods from conventional spectroscopic cells. The determination of first-generation fluorescence spectra of solid films represents a more significant challenge as the spectra in solution are not an accurate approximation to the spectra of the solid substance. For these structures, the first-generation spectra were studied by recording the spectra of fluorescence emitted from the front surface. An excitation angle of excitation of $70^{\circ}$ with respect to their surface normal was used in order to avoid a large amount of light reflected back to the detector and to illuminate a larger area of the collector. ${ }^{[11]}$ A $500 \mathrm{~nm}$ filter (Comar, 10 $\mathrm{nm}$ bandwidth) with a green LED (Luxeon, RS) or a Xenon arc lamp (AMKO, $75 \mathrm{~W}$ ) was used as the excitation light. A range of samples with decreasing dye concentration in the film were analysed to study the shape of the front fluorescence spectrum. It was found that for samples with absorbance below 0.07 the fluorescence emission spectra are independent of the sample absorbance. These spectra were equated to the true dye spectra free from re-absorption, and used as the first generation fluorescence spectra for 


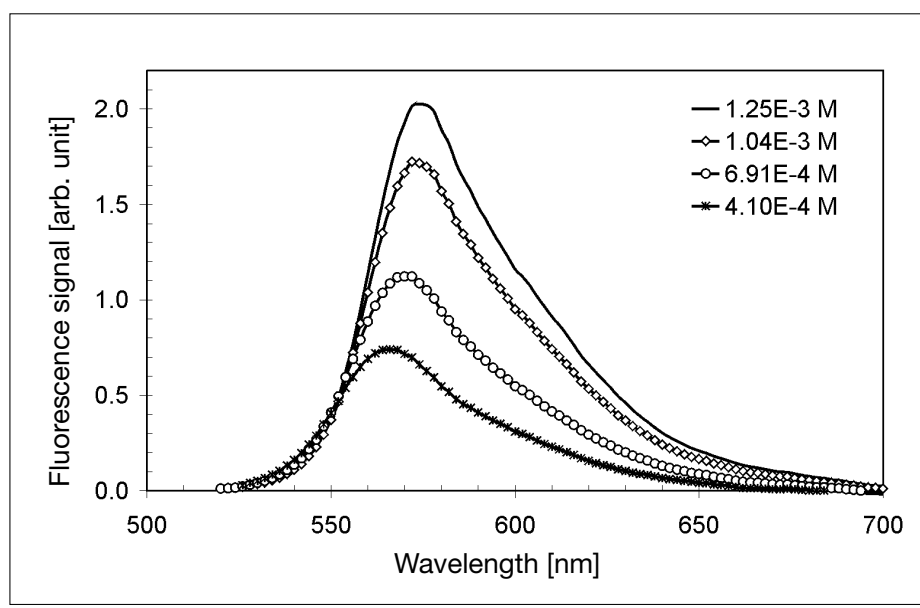

Fig. 4. Typical edge fluorescence spectra of Rhodamine $6 \mathrm{G}$ collectors. Numbers in legend give the approximate dye concentrations in the film layer.

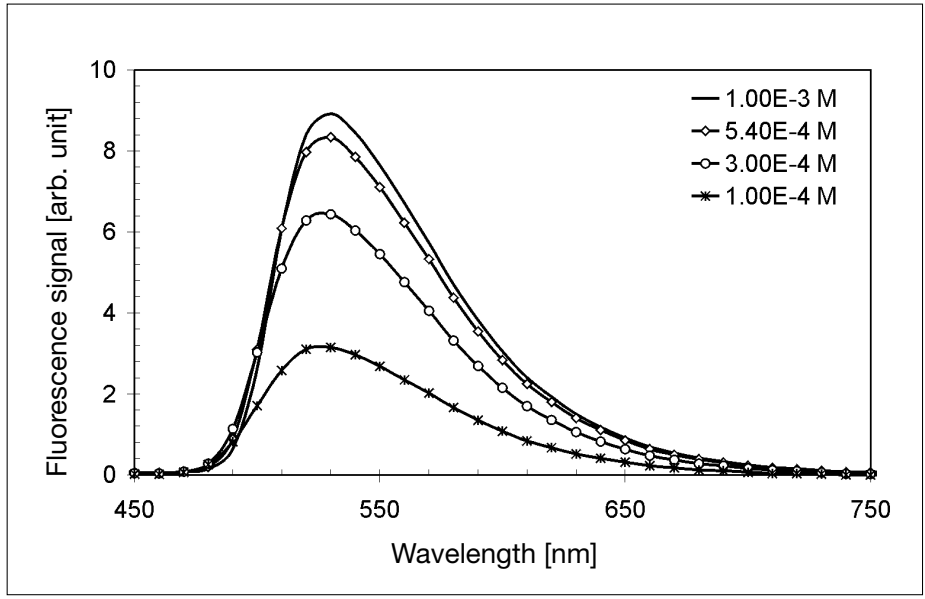

Fig. 5. Edge fluorescence spectra of liquid Coumarin 540A collectors. Legend gives the dye concentration in ethanol. the analysis in the following section. This procedure was confirmed using liquid collectors where the first generation spectra can be obtained reliably by conventional spectroscopy, as already noted. ${ }^{[11]}$

The fluorescence spectrum emitted from the edge of the collectors was collected by an optical fibre terminated with a cosine corrector (Avantes) to collect light over a large solid angle, eliminating optical interface problems associated with the light collection sampling geometry inherent to the optical fibre. The remaining edges were covered with a highly reflecting film (3M Vikuiti Enhanced Specular Reflector) with specular reflectance over $98 \%$ across the visible spectrum. This arrangement creates a setup equivalent to the infinite strip geometry and allows a comparison to be made with the results of Weber and Lambe. ${ }^{[1]}$ All the fluorescence spectra measured were shape corrected against the spectrum of a standard halogen lamp (Bentham Instruments Ltd).

\subsection{Solar Cell Measurements}

A solar simulator (T.S. Space Systems) equipped with a Xenon lamp approximating AM1.5 spectrum and a filter selection wheel (400-1100 nm, step $50 \mathrm{~nm}$ ) was used to measure the output of the fluorescent collectors. The photon collection efficiency $\left(Q_{C}\right)$ of the Rhodamine $6 \mathrm{G}$ collectors was measured at $500 \mathrm{~nm}$ by placing a silicon solar cell at one of the edges of the collector $(26 \mathrm{~mm} \times 26 \mathrm{~mm} \times 10 \mathrm{~mm})$ coupled with an optical gel (Thorlabs). The other three edges of the collector were covered with a highly reflective film. The cell was partially covered with black insulating tape to leave an area of $4 \times 26 \mathrm{~mm}^{2}$ available for illumination. The same cell was also illuminated directly from the $500 \mathrm{~nm}$ filter. Since the quantum efficiency of the cell is practically wavelength independent in this part of the spectrum, the measured short circuit current ratio of the collector/direct illumination gives the optical efficiency and hence $Q_{C}$.

\section{Results and Discussion}

Typical edge fluorescence spectra of fluorescent collectors with a single type of dye are shown in Figs. 4 and 5. Fig. 4 shows the results for a range of solid collectors with different concentrations of Rhod- amine 6G in PMMA films spin coated on glass slides. Fig. 5 corresponds to liquid collectors with different concentrations of Coumarin 540A in ethanol. Curves in each graph were obtained under identical illumination conditions, and each graph therefore corresponds to the same photon flux incident on the collector.

Before embarking on a detailed analysis of these results, a brief qualitative discussion is in place. Fig. 6 shows the edge fluorescence data of Figs. 4 and 5 but now

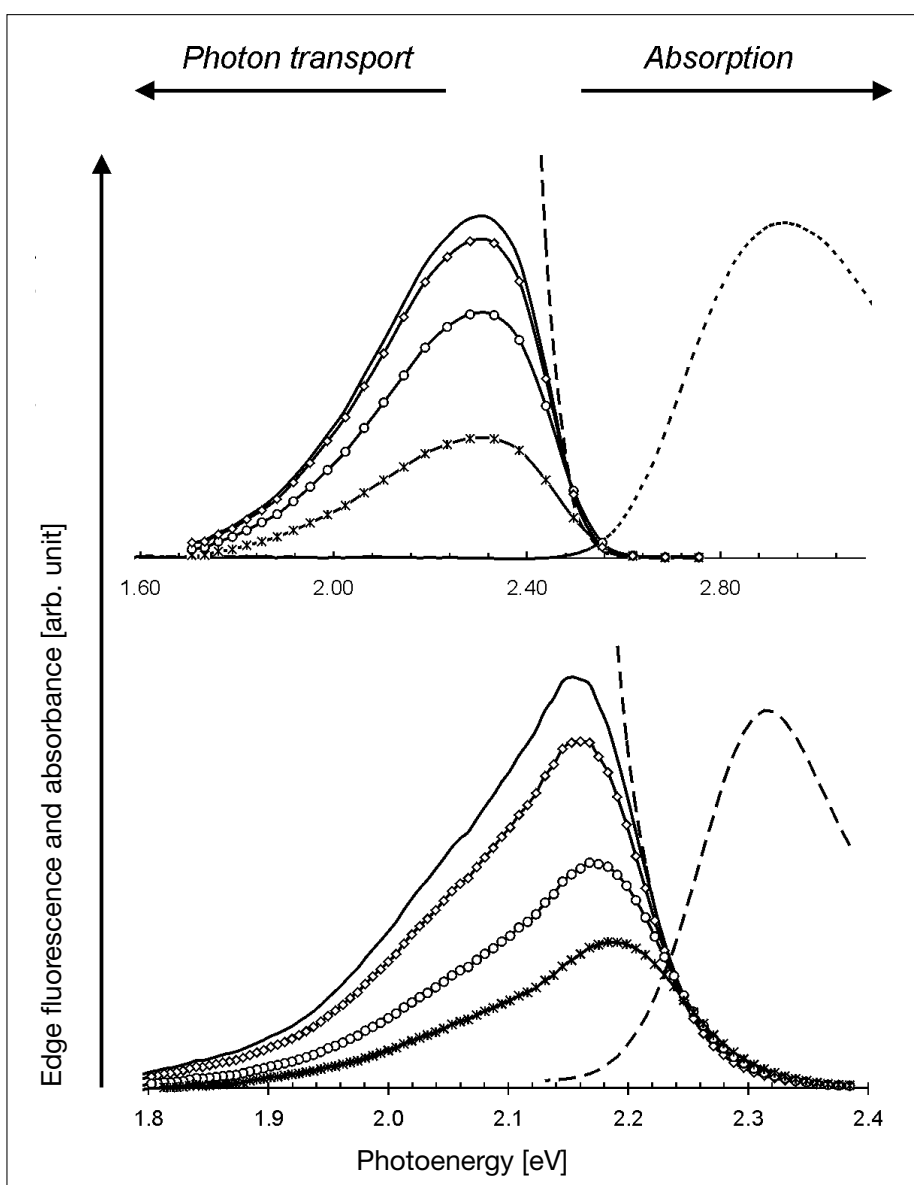

Fig. 6. The edge fluorescence spectra of Figs. 4 and 5 replotted as function of the photon energy for fluorescence collectors based on Coumarin 540A (top) and Rhodamine $6 \mathrm{G}$ (bottom). The dashed line shows the photon flux in thermal equilibrium with the collector; the dotted line gives the normalised absorbance. 


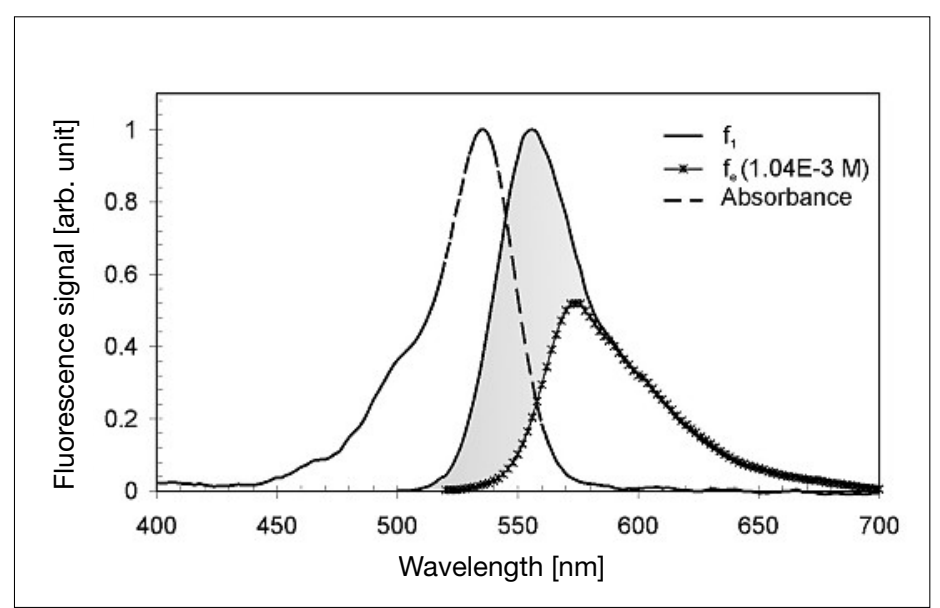

Fig. 7. Edge fluorescence spectra normalised to coincide with the first generation fluorescence spectrum $f_{1}$ in the low-absorption long-wavelength region. The shaded area is equal to the probability of re-absorption $R$.

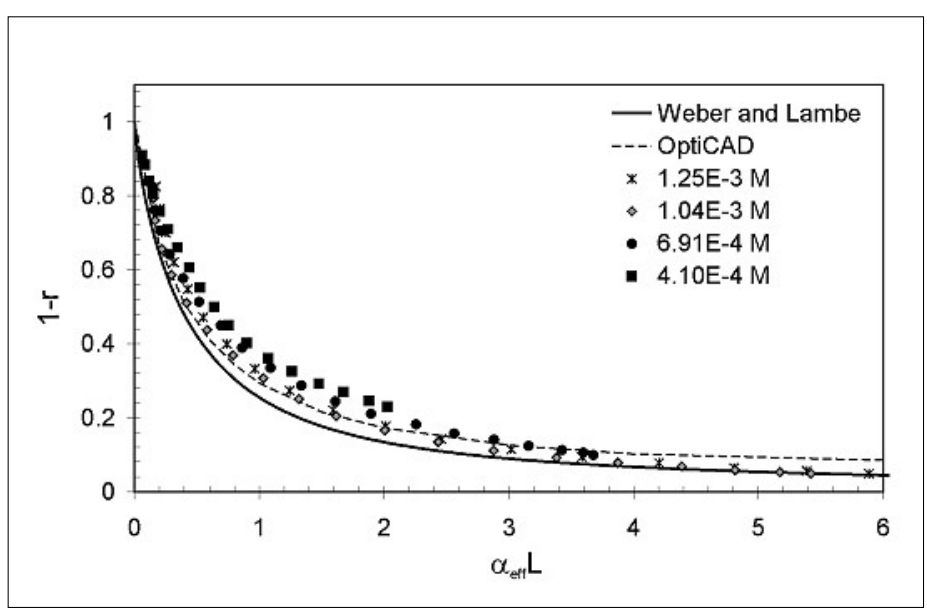

Fig. 8. The probability $1-r(\lambda)$ as a function of $\alpha_{\text {eff }} L$ as functions of photon energy, with the relevant absorbance graphs. Two characteristic spectral regions of collector operation now become apparent: the short-wavelength blue edge of the fluorescence spectrum characterised by high optical absorption, to be contrasted with the long-wavelength red edge where light can pass freely through the collector without significant re-absorption. We shall see in Section 5 that these two facets of photon transport in relation to absorption express the key features of collector operation, conveying important clues about how the collector performance can be improved.

Since the optical path length of photons in the high absorption region is shorter than the re-absorption length $\sim \alpha_{e m}{ }^{-1}$, photons will undergo multiple absorptions and reemissions before leaving the collector. As a result, the spectra are practically independent of dye concentration and, therefore, independent of the amount of light absorbed by the collector. For a near-unit fluorescence yield, the absorption-emission event is analogous to scattering between different quantum states. This somewhat surprising result can be understood by a simple analogy with gas kinetics where the absorption length for the emitted photons $\alpha_{e m}^{-1}$ takes the role of the mean free path. Since the amount of light absorbed from the incident beam is proportional to $1-\mathrm{e}^{-\alpha_{e x c} \mathrm{~d}} \approx \alpha_{e x c} d$, where $\alpha_{\text {exc }}$ is the absorption coefficient at the excitation wavelength and $d$ is the thickness of the collector, the amplitude of the emission spectrum is proportional to $\alpha_{\text {exd }}$ d $\alpha_{e m}$ which is independent of dye concentration.

Multiple absorptions by the dye with subsequent re-emission bring photons in this part of the spectrum to equilibrium with the fluorescent medium. One may therefore expect that the edge fluorescence spectra in this spectral region should resemble the spectrum of thermal radiation. This is indeed the case, as shown in Fig. 6 by the dashed lines which show the amplitude of thermal photon density, proportional to $E^{2} \exp (-E /$ $\left.k_{B} T^{*}\right)$, where $E$ is the photon energy and $k_{B}$ is the Boltzmann constant. The temperature $T^{*}$ of this radiation, however, is not always equal to the temperature of the collector. A more detailed analysis has shown that it is given by the Kennard-Stepanov theory of detailed balance between absorption and emission. More details of this aspect of fluorescent collector operation will be published in a separate publication. ${ }^{[12]}$

The situation is markedly different in the red (long wavelength) region where absorption is weak. Here, the typical optical path length inside the collector, which is traversed by emitted photons, is much longer than the absorption length. This part of the spectrum therefore corresponds to photons which are observed in the edge fluorescence with little or no re-absorption. One would therefore expect with the help of Eqn. (8) in Section 2 that, with appropriate normalisation, the shape of the edge emission spectrum in this spectral region be identical to the spectrum of first generation fluorescence. This is indeed the case. Fig. 7 shows that the edge fluorescence spectra of Fig. 4 which, when multiplied by an appropriate constant, coincide in the long wavelength region with the first generation fluorescence.

This procedure also represents a convenient method to determine the probability $1-r(\lambda)$, obtained by dividing this normalised edge fluorescence spectrum by $f_{1}$. The total probability of re-absorption $R$ can then be easily determined by integration, which has the graphical interpretation of an area between the first generation curve and the normalised edge fluorescence spectra (Fig. $7)$. The values of $1-r(\lambda)$ can then be plotted as a function of the absorption coefficient (or more precisely, $\alpha_{e f f}$ ) and compared with the Weber and Lambe curves. The results, shown in Fig. 8, are in good agreement with theory.

Our solar simulator measurements on a series of Rhodamine $6 \mathrm{G}$ collectors with ranging concentrations up to $2 \times 10^{-3} \mathrm{M}$ gave photon collection efficiencies $Q_{C}$ in the range of $45-50 \%$ - about $80-85 \%$ of the expected theoretical value for single dye collectors based on Rhodamine 6G. These high fluorescence collection efficiencies have been measured on simple collector structures and indicate that collector performances are possible near the theoretical values. On increasing the dye concentration further to $4 \times 10^{-3} \mathrm{M}$, the $Q_{C}$ value reduces significantly to less than $30 \%$, indicating fluorescence quenching which occurs at high concentrations associated with Rhodamine 6G. ${ }^{[13]}$ Preliminary measurements on Coumarin 540A solid collectors did not show this fluorescence quenching at high concentrations. The collectors here manifest even higher $Q_{C}$ values of about $67 \%$ which are maintained with increasing dye content.

\section{Outlook: Towards the Next Generation of Fluorescent Collectors}

Section 4 has given an overview of fluorescent collector operation based on a single dye. We have shown that, despite their simple structure, such collectors may reach high values of photon collection efficiencies. Their performance, however, as measured by the overall optical efficiency $\eta$, will be limited by low values of the absorption efficiency $Q_{A}$. No attempt is made here to discuss the experimental values of $Q_{A}$ under broad band illumination; instead, we turn to literature and modelling to assess the future potential which can be achieved by using several dyes to improve the coverage of the solar spectrum. ${ }^{[14-16]}$ 
A number of attempts have been made to report the total optical efficiency in the literature, with results generally in the order of few percent (see, for example, ${ }^{[15]}$ ). Such efficiencies are unlikely to be high enough for large-scale practical exploitation. Because of the intimate relationship between $Q_{A}$ and $Q_{C}$ through re-absorption it is also unlikely that higher optical efficiencies will be reached without a concerted strategy with respect to the choice of fluorescent dyes and their physical arrangement in the collector.

We shall show, by analysis of results discussed in Section 4 and modelling, that a significant increase in efficiency can be obtained by a judicious use of photon management and down shifting of the luminescent energy, achievable by a collection of dyes and/or dyes with several electronic states. One vehicle for the re-distribution of energy between the molecular states is re-absorption with the resulting loss of photons by emission within the escape cone. However, other - potentially more efficient - means of non-radiative energy transfer exist and could be applied to advantage (see, for example, ${ }^{[17]}$ ). Just like in conventional fluorescence spectroscopy, fluorescent emission in the collector will then occur from energy levels in thermal equilibrium, as we have already observed in the edge fluorescence spectra discussed in Section 4 in the limit of high re-absorption.

An efficient collector represents an optimum between a high absorption efficiency $Q_{A}$ and high collection efficiency $Q_{C}$. The absorption region therefore has to cover a large part of the solar spectrum, leaving a narrow photon transport region above the bandgap $E_{G}$ of the photovoltaic material to allow efficient photon transport to the solar cell. The spectral characteristics of the collector will therefore have the form shown schematically in Fig. 9, where $\Delta E$ denotes the width of the photon transport region in

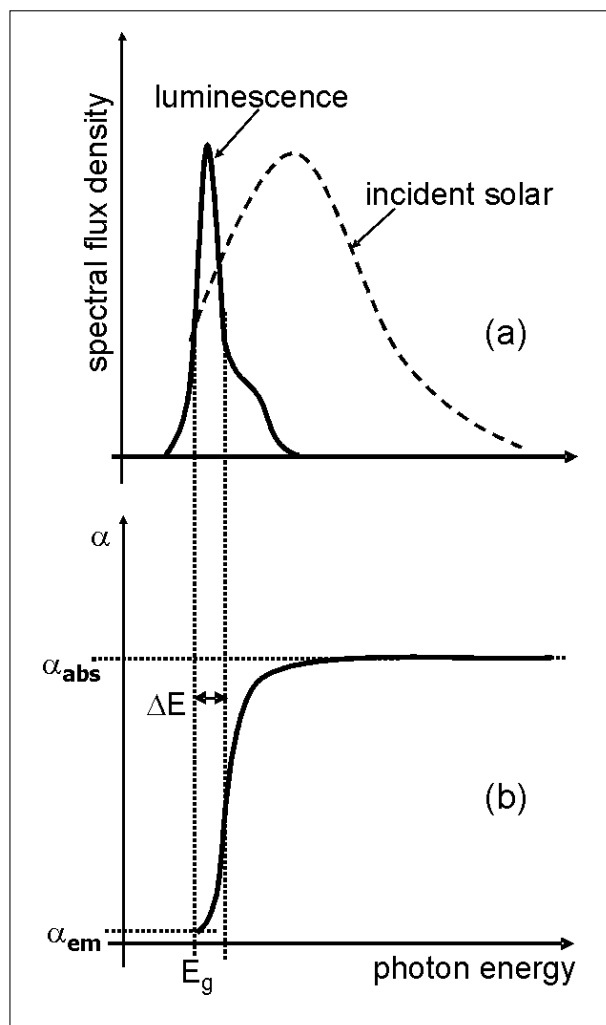

Fig. 9. Schematic diagram of the incident and emitted fluxes (a) and the absorption spectrum (b) for an optimum fluorescent collector with light trapping by TIR

energy units. Clearly, the absorption coefficients must satisfy

$$
\alpha_{a b s} \geq 1 / d ; \quad \alpha_{e m}<<1 / L
$$

for optimum operation of the collector of width $L$ and thickness $d$, as already defined in Section 2.

As in the single dye collectors, a fraction $P$ of photons in the photon transport band will be emitted in the escape cone and lost through the front face of the collector. Further losses will occur through re-absorption by activation to the absorption spectral band. The bandwidth $\Delta E$ of the photon transport region - which plays here the role of a generalised Stokes shift therefore defines a compromise between an optimum product of $Q_{\mathrm{A}}$ and $Q_{C}$. A large $\Delta E$ means a low probability of re-absorption and a high $Q_{C}$, but a narrower absorption band with lower $Q_{A}$. A small $\Delta E$ means a wider absorption region (high $Q_{A}$ ) but an increased probability of re-absorption, indicating a lower $Q_{C}$

A simple model can be constructed based on re-absorption proportional to the probability of thermally activated fluorescence emitted from the absorption band. Use can also be made of the kinetic nature of photon escape from the collector within the absorption band, as already noted in Section 4 . The approximate but simple result for $Q_{C}$ is ${ }^{[12]}$

$$
Q_{C}=\frac{1-P}{1+\frac{G}{2 n^{2} \alpha_{e m} L} e^{-\Delta E / k_{B} T}}
$$

where $T$ is the temperature of the collector, assumed to be equal to the KennardStepanov temperature $T^{*}$.

The results obtained by modelling for a geometrical gain of 26 (same as the solid collectors discussed in Section 4) are shown in Fig. 10. The best optical efficiency of approximately $58 \%$ would give total conversion efficiency exceeding $10 \%$ when coupled with a good crystalline silicon solar cell (Fig. 11). This figure takes into account the increase in open circuit voltage produced by an increase in intensity of light incident on the solar cell compared with the solar spectrum. The fluorescent collectors are then likely to attract interest in commercial operation in sizable systems

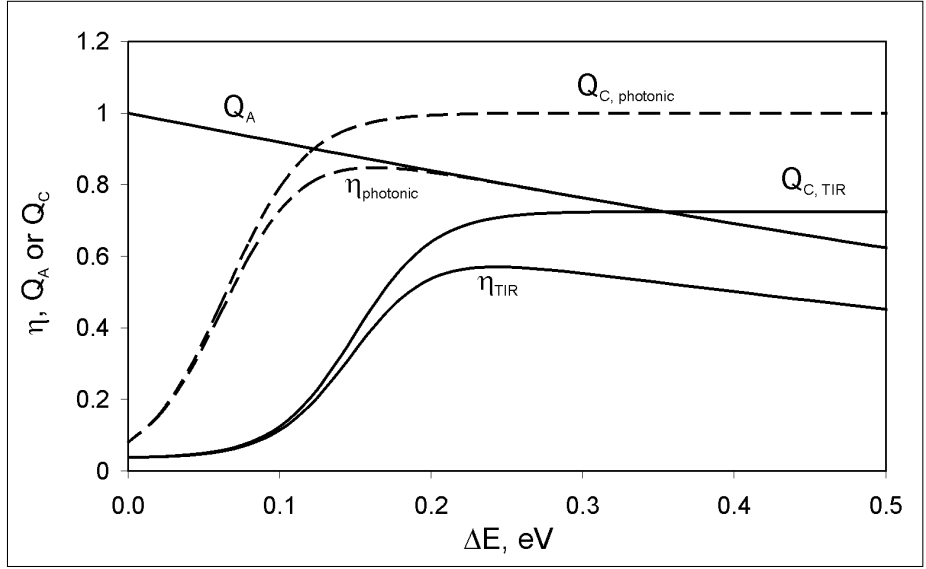

Fig. 10. The optical efficiency $\eta$, photon collection efficiency $Q_{C}$ and the absorption efficiency $Q_{A}$ for an optimum collector based on TIR (full lines) and a collector with photonic optical confinement (dashed lines). In both cases, the geometric gain is 26 .

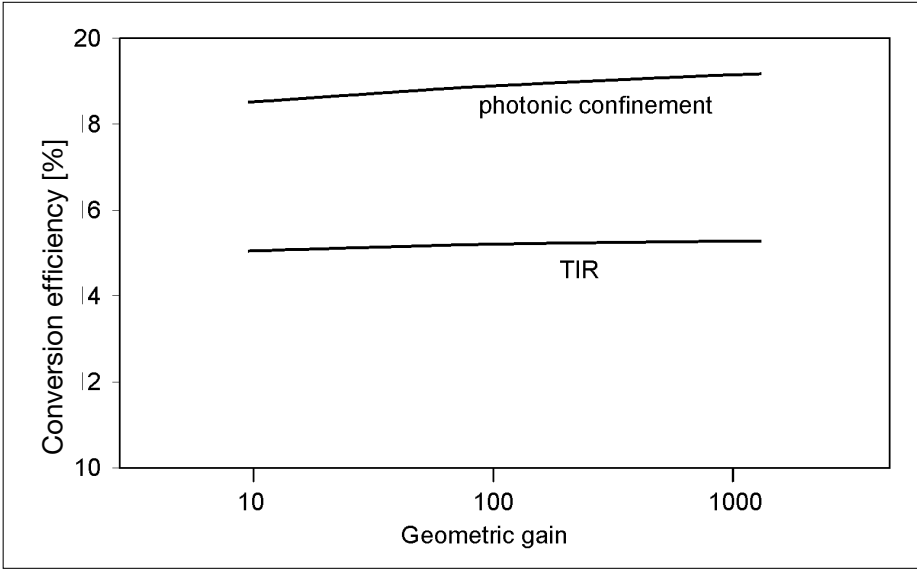

Fig. 11. The overall conversion efficiency of a collector with efficiencies described in Fig. 10, illuminating a $20 \%$ efficient crystalline silicon solar cell with short circuit current density (at one sun) $37.5 \mathrm{~mA} / \mathrm{cm}^{2}$, open circuit voltage $0.67 \mathrm{~V}$ and fill factor 0.8 
where $10 \%$ is generally considered to be the lowest target efficiency to avoid area related losses. ${ }^{[18]}$ We should note, however, that these efficiencies will only be achievable with values of the ratio $\alpha_{a b s} / \alpha_{\mathrm{em}}$ in the region of 2,000-3,000 and larger still for larger gain factors $G$ of the collector.

These figures probably represent the ultimate efficiency limits of collectors where light is trapped by total internal reflection. An improvement over this efficiency can only be obtained by eliminating photon losses through emission within the escape cone. The optical confinement then needs to rely on spectral selection rather than on geometric methods, and can be achieved with a spectrally selective mirror placed at the front face of the collector which reflects all photons with energy $E_{G}+\Delta E$. For such a collector the ultimate collection efficiency is given by, for a unit fluorescence quantum yield ${ }^{[19]}$

$$
Q_{C}=\frac{1}{1+\frac{G}{n^{2}}\left(\frac{E_{G}+\Delta E}{E_{G}}\right)^{2} e^{-\Delta E / k_{B} T}}
$$

Eqn. (13) shows that the photon collection efficiency is independent of the absorption coefficient which, however, is assumed to be sufficiently high for the collector to absorb all light in the entire spectral region of its operation. Figs. 10 and 11 show the results for such a collector by the dashed lines. We note that the use of such collector results in less than $10 \%$ relative loss of the solar cell output, in return for more than an order of magnitude saving in the amount of semiconductor material.

The operation of such devices represents the limit of fluorescent collector operation imposed by the second law of thermodynamics ${ }^{[12,19-22]}$, with photon losses reduced to levels which stem from microscopic reversibility. The collector then acts as an ideal converter of black body radiation, with optical efficiency determined by a detailed balance, similar to the argument used by Shockley and Queisser to determine the ultimate efficiency limits to the operation of solar cells. ${ }^{[23]}$ On this basis, it is not unrealistic to conjecture that the efficiency of collectors fabricated in the laboratory may one day reach $80 \%$.

Received: October 22, 2007
[1] W. H. Weber, J. Lambe, Appl. Opt. 1976, 15, 2299.

[2] J. A. Levitt, W. H. Weber, Appl. Opt. 1977, 16, 2684.

[3] A. Goetzberger, W. Greubel, App. Phys. 1977, 14, 123.

[4] C. F. Rapp, N. L. Boling, Proc. 13th IEEE Photovoltaic Specialists Conference, Berlin, Germany, 1978, pp. 690-693.

[5] J. S. Batchelder, A. H. Zewail, T. Cole, Appl. Opt. 1979, 18, 3090.

[6] J. S. Batchelder, A. H. Zewail, T. Cole, Appl. Opt. 1981, 20, 3733.

[7] A. M. Hermann, Solar Energy 1982, 29, 323.

[8] R. Reisfeld, C. K. Jorgensen, Structure and Bonding 1982, 49, 1.

[9] L. Danos, P. Kittidachachan, T. J. J. Meyer, R. Greef, T. Markvart, 'Proc. 21st European Photovoltaic Solar Energy Conference', Dresden, Germany, 2006, pp. 443-446.

[10] J. E. Parrott, Sol. Energy Mater. Sol. Cells 1993, 30, 221.

[11] See, for example, J. R. Lakowicz, 'Principles of Fluorescence Spectroscopy', 3rd ed., Springer, 2007.

[12] T. Markvart, Second Czech Renewable Energy Conf., Brno, 2006, to be published.

[13] P. Bojarski, Chem. Phys. Lett. 1997, 278, 225.

[14] B. A. Swartz, T. Cole, A. H. Zewail, Opt. Lett. 1977, 1, 73.

[15] P. S. Friedman, C. R. Parent, Solar Energy Research Institute Report SERI/ STR-211-3149, 1984

[16] S. T. Bailey, G. E. Lokey, M. S. Hanes J. D. M. Shearer, J. B. McLafferty, G. T. Beaumont, T. T. Baseler, J. M. Layhue, D. R. Broussard, Y. Zhang, B. P. Wittmershaus, Sol. Energy Mater. Sol. Cells 2007, 91, 67.

[17] G. Calzaferri, M. Pauchard, H. Maas, S. Huber, A. Khatyr, T. Schaafsma, J. Mater. Chem. 2002, 12, 1.

[18] 'Practical Handbook of Photovoltaics: Fundamentals and Applications', Eds. T. Markvart, L. Castañer, Elsevier, Oxford, 2003.

[19] T. Markvart, J. Appl. Phys. 2006, 99, 026101; T. Markvart, Appl. Phys. Lett. 2006, 88, 176101.

[20] E. Yablonovich, J. Opt. Soc. Am. 1980, 70, 1362.

[21] U. Rau, F. Einsele, G.C. Glaeser, Appl. Phys. Lett. 2005, 87, 171101; U. Rau, F. Einsele, G.C. Glaeser, Appl. Phys. Lett. 2006, 88, 176102.

[22] T. Markvart, 'Thermodynamics of optical étendue', to appear in J. of Opt. A: Pure and Applied Optics.

[23] W. Shockley, H.J. Queisser, J. Appl. Phys. 1961, 32, 510 . 